Method A design collaboration including a specialist MRI small-medium enterprise, clinicians, physicists and national metrology institutes was formed. A T2 mapping phantom (figure 1 i) was designed to cover clinically relevant T1 and T2 times in native and post-contrast myocardium across fieldstrengths (figure 1 iii,v). Two earlier prototypes had been manufactured and tested, with the third and final one being reported here.

Results The T2 mapping phantom which can be used at both 1.5 and 3 Tesla is an agarose gel-based phantom using nickel chloride as the paramagnetic relaxation modifier. It contains nine differently-doped agarose gel tubes embedded in a gel/ beads matrix.

The phantom was free of air bubbles and susceptibility artifacts at both field strengths (figure 1 ii) and T2 maps were free from off-resonance artifacts (figure 1 iv). The incorporation of high-density polyethylene beads in the main gel fill was effective at flattening the $B_{0}$ and $B_{1}$ fields (figure $2 \mathrm{i}$,ii). T1 and T2 times measured in the phantom showed coefficients of variation of $\leq 1 \%$ between repeat scans indicating good short-term reproducibility. Temperature dependency experiments conducted at the national metrology institutes
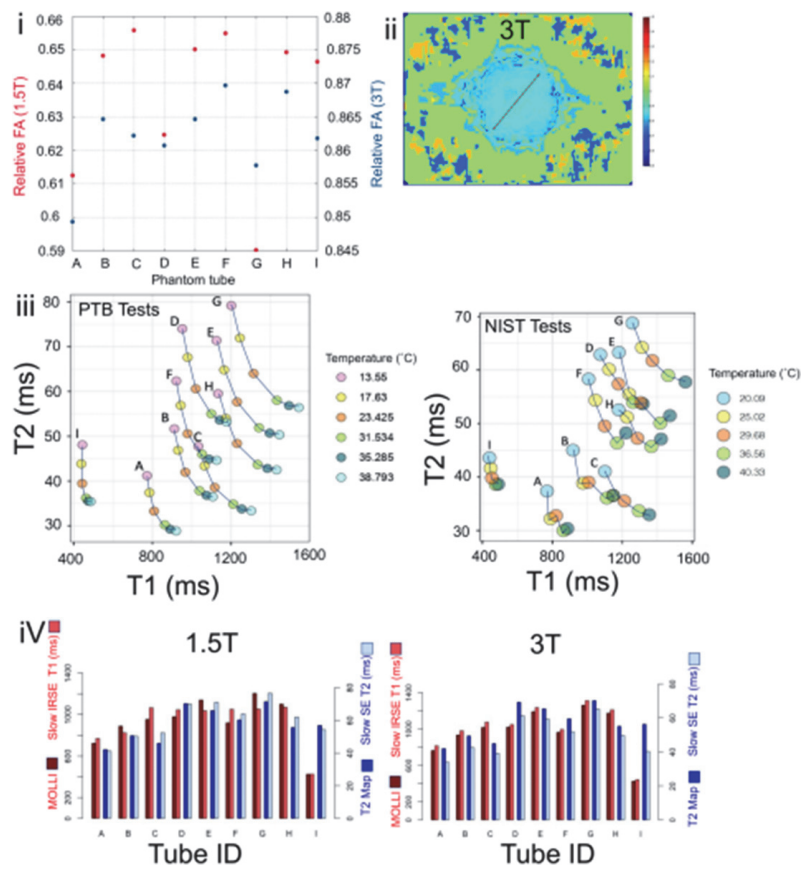

Abstract 11 Figure 2 (i) $B_{0}$ field homogeneity across the nine phantom compartments as a measure of off-resonance in hertz at $1.5 \mathrm{~T}$ (red) and $3 \mathrm{~T}$ (blue). These are extremely small shifts in frequency (e. g., $10 \mathrm{~Hz}=0.08 \mathrm{ppm}$ at $3 \mathrm{~T}$ ) and should not be regarded as significantly different between the tube compartments. (ii) T2 phantom field map at 3T showing $B_{1}$ homogeneity across the device cross-section. (iii) Temperature tests carried out at PTB-German Physikalisch-Technische Bundesanstalt (Left) -using a 3T Siemens Magnetom Verio system (VB17) and a 12-channel head coil and at NIST-US National Institute of Standards and Technology (Right) - using Agilent 3T small bore scanner. T1 was measured by IRSE (TR $[\mathrm{s}]=10, \mathrm{TI}[\mathrm{ms}]=50,75,100$, $125,150,250,500,1000,1500,2000,3000,6000)$ and T2 by SE (TR $[\mathrm{s}]=10, \mathrm{TE}[\mathrm{ms}]=14,28,56,112,224)$; resolution: $0.5 \times 0.5 \mathrm{~mm}$; slice thickness: $2 \mathrm{~mm}$. (iv) Comparison of reference T1|T2 times (by IRSE/SE respectively) to those obtained by MOLLI (5(3)3) and T2 mapping (SSFP) at 1.5T (Siemens Aera operating VE11C) and 3T (Siemens Prisma operating VE11C). TE=echo time; TR=repetition time. Other abbreviations as in figure 1. (figure 2 iii) confirmed that over the range $13-40^{\circ} \mathrm{C}$ the short-T1/2 tubes were more stable with temperature than the long-T1/2 tubes.

Conclusion The program has developed a T2 mapping phantom for CMR replicating clinically relevant T1/T2 times across myocardial health and disease. The device will be shortly listed under the Food and Drug Administration (FDA) database and Conformité Européene (CE) marking. Reproducible mass manufacture of this phantom may now commence to support the use of T2 mapping in longitudinal cohort studies, multicentre research or inflammation imaging.

\section{MYOCARDIAL INFLAMMATION AND DIFFUSE FIBROSIS UNDERPIN THE ELECTROPHYSIOLOGICAL DERANGEMENTS OF THE AGEING HUMAN HEART-A CMR-ECGI STUDY}

1,2,3,4 Matthew Webber, ${ }^{1,2}$ George Joy, ${ }^{3}$ Debbie Falconer, ${ }^{5}$ Xuyuan Tao, 1,2 lain Pierce, ${ }^{1,2}$ James C Moon, ${ }^{2,4}$ Alun D Hughes, ${ }^{1,2}$ Pier D Lambiase, ${ }^{6,7}$ Yoram Rudy* ${ }^{2,4}$ Michele Orini* ${ }^{*}, 3,4$ Gabriella Captur. ${ }^{1}$ Barts Heart Centre, Barts Health NHS Trust, West Smithfield, ECIA 7BE, London, UK; ${ }^{2}$ Institute of Cardiovascular Science, University College London, Huntley Street, WC1E 6DD, London, UK; ${ }^{3}$ Centre for Inherited Heart Muscle Conditions, Department of Cardiology, Royal Free London NHS Foundation Trust, Pond Street, NW3 2QG, London, UK; ${ }^{4}$ Medical Research Council Unit for Lifelong Health and Ageing at UCL, 1-19 Torrington Place, WC1E 7HB, London, UK; ${ }^{5} E$ cole Nationale Supérieure des Arts et Industries Textiles, 2 Allée Louise et Victor Champier, 59056 Roubaix CEDEX 1, France; ${ }^{6}$ Cardiac Bioelectricity and Arrhythmia Center, Washington University, St Louis, MO 63130, USA; ${ }^{7}$ Department of Biomedical Engineering, Washington University, St. Louis, MO 63130, USA

10.1136/heartjnl-2021-BSCMR.12

Background Susceptibility to malignant ventricular arrhythmias can be predicted by activation recovery interval (ARI) and repolarisation time (RT) biomarkers noninvasively derived by electrocardiographic imaging (ECGI). The interaction between cardiac structural and electrophysiological changes during aging is unclear. We used our recently developed cardiovascular magnetic resonance (CMR)-ECGI vest to understand how the ageing myocardial substrate assessed by CMR interacts with these ECGI biomarkers under complete physiological conditions.

Methods CMR-ECGI at 3 Tesla was prospectively performed (figure 1). 256-Lead body surface potentials were recorded and co-registered with CMR-derived heart-torso geometries. Epicardial unipolar potentials were reconstructed using stateof-the-art ECGI algorithms to derive whole-heart electrophysiological maps (figure 2).

Results 45 Participants were recruited: 29 older persons from a population-based cohort (all 75 years; $55 \%$ male) and 16 young healthy volunteers ( $34 \pm 3$ years, 62\% male). Compared to young participants, older ones showed longer T1 $(1300 \pm 38.4 \mathrm{~ms}$ vs $1280 \pm 46.5 \mathrm{~ms}, p=0.020)$ and $\mathrm{T}_{2}$ $(46.1 \pm 0.06 \mathrm{~ms}$ vs $42.9 \pm 2.3 \mathrm{~ms}, p=0.001)$ and higher extracellular volume (ECV, 26.6 $\pm 1.9 \%$ vs $22.6 \pm 3.5 \%$, $p<0.001)$. Activation time (AT) was prolonged in older compared to younger hearts $(41.8 \pm 19.7 \mathrm{~ms}$ vs $36.8 \pm 20.0$ $\mathrm{ms}, p=0.042$ ) with similar but weaker trends observed for RT and ARI $(p=0.086, p=0.160)$. Apart from left ventricular ejection fraction (LVEF, e.g. for ARI $\beta=1.9 \mathrm{~ms}[0.01-$ 3.7] $p=0.049$ ), no other standard CMR size/function parameter or binary presence of late gadolinium enhancement, showed an association with ECGI parameters. By contrast, native $\mathrm{T}_{1}, \mathrm{~T}_{2}$ and $\mathrm{ECV}$ were all associated with $\mathrm{RT}$ 


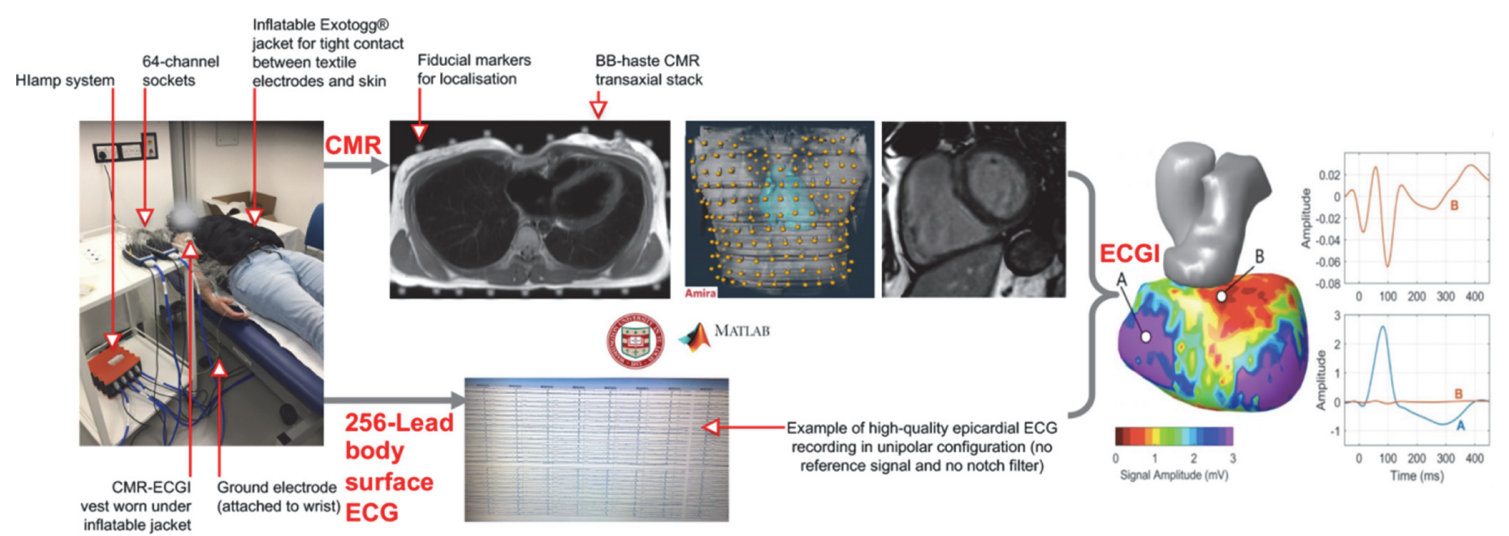

Abstract 12 Figure 1 Agile CMR-ECGI workflow. ECGI recording precedes CMR using Hlamp multi-channel amplifier connected to g.Recorder software at a sampling frequency of $2048 \mathrm{~Hz}$, for 5 mins at rest in the supine position. CMR-ECGI vest montage and doffing is quick with total preparation time per patient $<5$ mins, thanks to the vest design and dry electrode solution. CMR protocol includes transaxial black blood (BB) anatomical stack for electro-anatomical mapping using fiducial markers for co-registration. Electrode-skin surface contact is enhanced using an inflatable jacket. An exemplar activation time isochrone map is shown for a 75-year study member with midwall late gadolinium enhancement in the mid anteroseptal: note the matching zone of fractionation and low-amplitude in the unipolar electrograms. CMR=cardiovascular magnetic resonance; $\mathrm{ECGI}=$ electrocardiographic imaging.

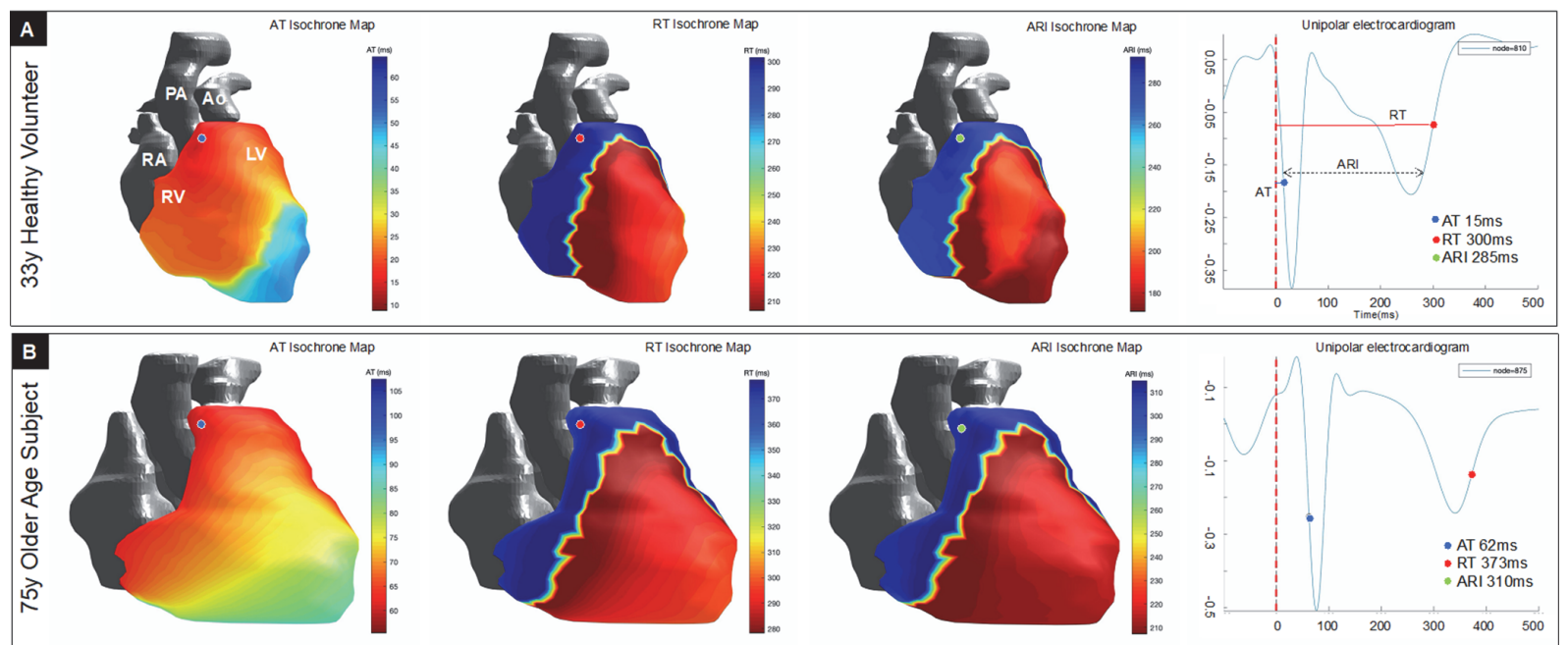

Abstract 12 Figure 2 Activation and recovery time isochrone maps arising from CMR-ECGI in a healthy 33-year old female volunteer (Top panel A) and in a 75-year-old male with no significant past medical history (Bottom panel B). Unipolar electrocardiograms are shown for each participant representing the point of initial activation. Of note is the prolonged activation time, repolarisation time and activation recovery interval of the older participant when compared to the young control. ARI=activation recovery interval; AT=activation time; RT=repolarisation time; $y=y e a r s$.

prolongation (respectively $\beta=0.3 \mathrm{~ms}[95 \%$ confidence interval $0.01-0.64] p=0.048 ; \beta=6.0 \mathrm{~ms}[1.85-10.05] p=0.006$; $\beta=4.9$ ms $[0.22-9.60] p=0.041)$ and, $\mathrm{T}_{2}$ and ECV with ARI prolongation $(\beta=5.9 \mathrm{~ms}[1.88-9.92] p=0.005 ; \beta=4.8$ ms [0.40-0.12] $p=0.033)$. All associations persisted after adjusting for LVEF.

Conclusion The normal electrophysiological sequence of activation and repolarisation in the human heart changes markedly with ageing and may be explained by CMR-detected myocardial substrate changes consisting of low-grade inflammation and diffuse fibrosis. Our high-throughput and reusable CMR-ECGI solution has the ability to provide unprecedented insights into the pathophysiology of arrhythmogenesis beyond conventional measures of cardiac structure and function.

\section{THE ASSOCIATION BETWEEN CARDIOVASCULAR RISK FACTORS AND LEFT ATRIAL STRUCTURE AND PHASIC FUNCTION}

${ }^{1,2}$ Mihir M Sanghvi, ${ }^{1}$ Jose D Vargas, ${ }^{3}$ Avan Suinesiaputra, ${ }^{3}$ Rodrigo Bonazzola, ${ }^{3}$ Rahman Attar, ${ }^{3}$ Nishant Ravikumar, ${ }^{1}$ Mark Wong, ${ }^{1}$ Hafiz Naderi, ${ }^{1,2}$ Steffen E Petersen, ${ }^{3}$ Alejandro F Frangi, ${ }^{1,2}$ Nay Aung. ${ }^{1}$ Centre for Advanced Cardiovascular Imaging, William Harvey Research Institute, Queen Mary University of London, Charterhouse Square, London, EC1M 6BQ; ${ }^{2}$ Barts Heart Centre, Barts Health NHS Trust, West Smithfield, London, EC1A 7BE; ${ }^{3}$ School of Computing, University of Leeds, Woodhouse, Leeds, LS2 9JT

\subsection{6/heartjnl-2021-BSCMR.13}

Introduction Changes in left atrial (LA) phasic function defined as reservoir function (filling), conduit function (passive emptying), booster function (active emptying) (figure 1A) are thought to precede structural changes (dilatation) of the 